\title{
Erratum to: Smooth extensions of functions on separable Banach spaces
}

\author{
D. Azagra - R. Fry • L. Keener
}

Published online: 1 September 2010

(C) Springer-Verlag 2010

Erratum to: Math. Ann. (2010) 347:285-297

DOI 10.1007/s00208-009-0441-6

We fill a gap in the proof of Theorem 1 in the original paper by providing a sharper version of Lemma 1 in that paper.

We will use the same notation as in that paper. In the second part of the proof of Theorem 1 [where one wants to show that if one requires $f$ to be Lipschitz on $Y$ then one can choose the approximate extension $g$ to be $\operatorname{Lipschitz}$ with $\operatorname{Lip}(g) \leq C \operatorname{Lip}(f)]$, we need to use the fact that there is a function $\delta_{k}: X \rightarrow \mathbb{R}$ such that

(1) $\left|T_{k}(x)-F(x)-\delta_{k}(x)\right|<2^{-i-2} \varepsilon M_{k}^{-1}$ for all $x \in B_{2 s_{k}}$

(2) $\left\|\delta_{k}^{\prime}(y)\right\|_{X^{*}}<\varepsilon / 8$ for all $y \in B_{2 s_{k}} \cap Y$, and

(3) $\operatorname{Lip}\left(\delta_{k}\right) \leq C_{0} \operatorname{Lip}\left(T_{k}-F\right) \leq 2 C_{0} \operatorname{Lip}(F)$.

The online version of the original article can be found under doi:10.1007/s00208-009-0441-6.

D. Azagra $(\varangle)$

ICMAT (CSIC-UAM-UC3-UCM), Departamento de Análisis Matemático,

Facultad Ciencias Matemáticas, Universidad Complutense, 28040 Madrid, Spain

e-mail: daniel_azagra@mat.ucm.es

R. Fry

Department of Mathematics and Statistics, Thompson Rivers University,

Kamloops, BC, Canada

e-mail: rfry@stfx.ca

L. Keener

Department of Mathematics and Statistics, University of Northern British Columbia,

Prince George, BC, Canada

e-mail: keener@unbc.ca 
In that proof we claimed that the existence of a function $\delta_{k}$ satisfying (1) and (2) was guaranteed by Lemma 1 . In fact the version of Lemma 1 in the paper gives the existence of a function $\delta_{k}$ satisfying (2), but property (1) only holds on $B_{2 s_{k}} \cap Y$ for this function [and it is no use trying to apply Lemma 1 to $T_{k}-F$ on $Y=X$ because that way we would obtain another function $\delta_{k}$ satisfying (1) and (3), but not (2)].

As well, the equation

$$
\operatorname{Lip}\left(\Delta_{i}\right) \leq \max \left\{M \operatorname{Lip}(f)+\frac{\varepsilon}{8}, C_{0} \operatorname{Lip}(f)\right\},
$$

on page 292 of the paper is wrong, as it should have read

$$
\operatorname{Lip}\left(\Delta_{i}\right) \leq M \operatorname{Lip}(f)+3 C_{0} \operatorname{Lip}(f) .
$$

This is a minor mistake since the latter is true as long as $\delta_{k}$ satisfies property (3) above.

Next we state and prove a sharp version of Lemma 1 of the original paper which allows one to find functions $\delta_{k}$ satisfying all three properties above, thus filling the gap in the proof of Theorem 1. The proof of this sharp version of Lemma 1 can be easily deduced from the version stated in the paper, which we give below.

Lemma 1 Let $X$ be a separable Banach space with a $C^{1}$ smooth norm. There exists a constant $C_{0} \geq 1$ such that, for every subspace $Y \subseteq X$, every Lipschitz function $f: X \rightarrow \mathbb{R}$, and every $\varepsilon>0$, there exists a $C^{1}$-smooth function $K: X \rightarrow \mathbb{R}$ such that

(1) $|f(x)-K(x)|<\varepsilon$ for all $x \in X$,

(2) $\operatorname{Lip}(K) \leq C_{0} \operatorname{Lip}(f)$, and

(3) $\left\|K^{\prime}(y)\right\|_{X^{*}} \leq C_{0} \operatorname{Lip}\left(f_{\left.\right|_{Y}}\right)$ for all $y \in Y$ (in particular the Lipschitz constant of the restriction of $K$ to $Y$ is of the order of the Lipschitz constant of the restriction off to $Y$ ).

Proof Let us denote $\eta=\operatorname{Lip}\left(f_{\left.\right|_{Y}}\right)$ and $L=\operatorname{Lip}(f)$. Obviously $\eta \leq L$.

Consider the function $\varphi: X \rightarrow \mathbb{R}$ defined by

$$
\varphi(x)=\inf \{f(y)+\eta\|x-y\|: y \in Y\},
$$

which is an $\eta$-Lipschitz extension of the restriction $f_{\left.\right|_{Y}}$ to the subspace $Y$. By the version of Lemma 1 in the paper applied to $\varphi$ with $Y=X$, there exists a $C \eta$-Lipschitz, $C^{1}$ function $g: X \rightarrow \mathbb{R}$ such that $|g-\varphi| \leq \varepsilon / 3$ on $X$. Similarly there exists a $C L$-Lipschitz, $C^{1}$ function $h: X \rightarrow \mathbb{R}$ such that $|h-f| \leq \varepsilon$ on $X$. Here $C$ is the constant (not depending on $f$ or $\varepsilon$ ) provided by the old statement of Lemma 1 in the paper.

Consider the quotient space $X / Y$. It is standard that $(X / Y)^{*}$ is isometric to the closed subspace $Y^{\perp}$ of $X^{*}$ (see e.g., [2, Proposition 2.7]), and since $X^{*}$ is separable then so is $(X / Y)^{*}$. Since in a space with a separable dual every equivalent norm can be uniformly approximated in the unit ball by $C^{1}$ smooth equivalent norms (see [1]), $X / Y$ has an equivalent $C^{1}$ smooth norm (which we will also denote $\|\cdot\|$ ) with the property that

$$
\operatorname{dist}(x, Y) \leq\|q(x)\| \leq 2 \operatorname{dist}(x, Y) \quad \text { for all } x \in X \text {. }
$$


In particular the function $x \mapsto\|q(x)\|$ is 2-Lipschitz on $X$, as is easily checked.

Let $r=\varepsilon / 6 L>0$, and let $\alpha: \mathbb{R} \rightarrow[0,1]$ be a $C^{1}$ function such that $\alpha(t)=1$ for $t \leq r, \alpha(t)=0$ for $t \geq 2 r$, and $\operatorname{Lip}(\alpha) \leq 2 / r=12 L / \varepsilon$. Define $\beta=1-\alpha$. Then $\{\alpha, \beta\}$ form a $12 L / \varepsilon$-Lipschitz, $C^{1}$ smooth partition of unity on $\mathbb{R}$ with $\operatorname{supp}(\alpha) \subset(-\infty, 2 r)$ and $\operatorname{supp}(\beta) \subset(r,+\infty)$.

Define a function $K: X \rightarrow \mathbb{R}$ by

$$
K(x)=g(x) \alpha(\|q(x)\|)+h(x) \beta(\|q(x)\|) .
$$

Note that if $\alpha(\|q(x)\|) \neq 0$ then $\|q(x)\|<2 r$, hence also dist $(x, Y)<2 r$, and there exists $y_{x} \in Y$ with $\left\|x-y_{x}\right\|<2 r$. Then, since $g$ is $\eta$-Lipschitz and $f$ is $L$-Lipschitz, $|g-\varphi| \leq \varepsilon / 3$ and $\varphi_{\left.\right|_{Y}}=f_{\left.\right|_{Y}}$, we have

$$
\begin{aligned}
\left|g(x)-g\left(y_{x}\right)\right| & \leq \eta\left\|x-y_{x}\right\| \leq \eta 2 r \leq \varepsilon / 3, \\
\left|g\left(y_{x}\right)-f\left(y_{x}\right)\right| & \leq \varepsilon / 3, \\
\left|f\left(y_{x}\right)-f(x)\right| & \leq L\left\|x-y_{x}\right\| \leq L 2 r \leq \varepsilon / 3,
\end{aligned}
$$

from which we get

$$
|g(x)-f(x)| \leq \varepsilon \text { whenever } \alpha(\|q(x)\|) \neq 0 .
$$

And we always have $|h-f| \leq \varepsilon$. Therefore we can estimate

$$
\begin{aligned}
|K(x)-f(x)| & =|(g(x)-f(x)) \alpha(\|q(x)\|)+(h(x)-f(x)) \beta(\|q(x)\|)| \\
& \leq|g(x)-f(x)| \alpha(\|q(x)\|)+|h(x)-f(x)| \beta(\|q(x)\|) \\
& \leq \varepsilon(\alpha(\|q(x)\|)+\beta(\|q(x)\|))=\varepsilon,
\end{aligned}
$$

which shows (1).

As for the Lipschitz constant of $K$, assuming $\alpha(\|q(x)\|) \neq 0$, we have

$$
\begin{aligned}
&|K(x)-K(z)| \\
&=|g(x) \alpha(\|q(x)\|)+h(x) \beta(\|q(x)\|)-g(z) \alpha(\|q(z)\|)-h(z) \beta(\|q(z)\|)| \\
&=\mid(g(x)-f(x))(\alpha(\|q(x)\|)-\alpha(\|q(z)\|)) \\
& \quad+(h(x)-f(x))(\beta(\|q(x)\|)-\beta(\|q(z)\|)) \\
& \quad+(g(x)-g(z)) \alpha(\|q(z)\|)+(h(x)-h(z)) \beta(\|q(z)\|) \mid \\
& \leq \sup \{|f(u)-g(u)|: \alpha(\|q(u)\|) \neq 0\} 2 \operatorname{Lip}(\alpha)\|x-z\| \\
& \quad+\sup \{|h(v)-f(v)|: v \in X\} 2 \operatorname{Lip}(\beta)\|x-z\| \\
& \quad+\operatorname{Lip}(g)\|x-z\| \alpha(\|q(z)\|)+\operatorname{Lip}(h)\|x-z\| \beta(\|q(z)\|) \| \\
& \leq 2 \varepsilon \operatorname{Lip}(\alpha)\|x-z\|+2 \varepsilon \operatorname{Lip}(\beta)\|x-z\| \\
&+\max \{\operatorname{Lip}(g), \operatorname{Lip}(h)\}(\alpha(\|q(z)\|)+\beta(\|q(z)\|))\|x-z\| \\
& \leq 2 \varepsilon\left(\frac{12 L}{\varepsilon}+\frac{12 L}{\varepsilon}\right)\|x-z\|+C L\|x-z\|=(48 L+C L)\|x-z\| .
\end{aligned}
$$


The same estimation holds in the case $\alpha(\|q(z)\|) \neq 0$ (just replace $x$ with $z$ and use the symmetry of the norm). And in the case $\alpha(\|q(x)\|)=0=\alpha(\|q(z)\|)$ we have $|K(x)-K(z)|=|h(x)-h(z)| \leq C L\|x-z\|$. In any case we see that

$$
|K(x)-K(z)| \leq(48 L+C L)\|x-z\| \quad \text { for all } x, z \in X,
$$

which shows (2) with $C_{0}:=48+C$.

Finally, since $K=g$ on the open neighborhood $\{x \in X: \operatorname{dist}(x, Y)<r / 2\}$ of $Y$, and $\operatorname{Lip}(g) \leq C \eta$, it is clear that

$$
\left\|K^{\prime}(y)\right\|_{X^{*}}=\left\|g^{\prime}(y)\right\|_{X^{*}} \leq C \eta \leq C_{0} \eta \quad \text { for all } y \in Y
$$

We finally note that for the proof of Theorems 4 and 5 of the original paper, where $Y \subset X$ is assumed to be (merely) a closed subset, that one can again employ the technique of the above Lemma 1 by simply replacing the function $x \rightarrow\|q(x)\|$ with a uniform approximation of the distance function to $Y$ by a Lipschitz, $C^{1}$ smooth map.

\section{References}

1. Deville, R., Godefroy, G., Zizler, V.: Smoothness and Renormings in Banach Spaces, vol. 64, Pitman Monographs and Surveys in Pure and Applied Mathematics (1993)

2. Fabian, M., Habala, P., Hájek, P., Montesinos, V., Pelant, J., Zizler, V.: Functional unalysis and infinitedimensional geometry. In: CMS Books in Mathematics, vol. 8. Springer, Berlin (2001) 